\title{
HEMATOMA ESPONTÁNEO DE FARINGOLARINGE Y ANTICOAGULACIÓN. DESCRIPCIÓN DE UN CASO
}

\section{Spontaneous pharyngo-laryngeal hematoma and anticoagulation. A case report}

\author{
Marleny CASASOLA-GIRÓN; José Ignacio BENITO-OREJAS*; María Antonia CARRANZA- \\ CALLEJA; Ana SÁNCHEZ-MARTÍNEZ; Darío MORAIS-PÉREZ
}

SACYL. Hospital Clínico Universitario de Valladolid. Servicio de Otorrinolaringología y Patología Cervicofacial. Valladolid. España.

*Correspondencia: jbenito@ono.com

Fecha de recepción: 1 de noviembre de 2015

Fecha de aceptación: 5 de noviembre de 2015

Fecha de Publicación: 14 de enero de 2016

Conflicto de intereses: Los autores declaran no tener conflictos de intereses

Imágenes: Los autores declaran haber obtenido las imágenes con el permiso de los pacientes

Política de derechos y autoarchivo: se permite el autoarchivo de la versión post-print (SHERPA/RoMEO)

Licencia CC BY-NC-ND. Licencia Creative Commons Atribución-NoComercial-SinDerivar 4.0 Internaciona

(C) Universidad de Salamanca. Su comercialización está sujeta al permiso del editor

RESUMEN

Introducción y objetivo: El hematoma espontáneo faringolaríngeo pone nuevamente de manifiesto la importancia de realizar una completa exploración ORL ante síntomas de apariencia banal y una correcta anamnesis, que en el caso presentado, desveló el uso terapéutico de anticoagulantes. Descripción del caso: Una mujer de 55 años acude a urgencias por disfagia, sin causa aparente. La inspección muestra la presencia de un hematoma en la región faringolaríngea, que tras revertir la terapia anticoagulante, evolucionó favorablemente con tratamiento conservador. Discusión: En este caso, además del manejo médico que realice el Servicio de Hematología, debemos centrar nuestra actitud terapéutica, en la protección de la vía aérea y en prevenir una posible hemorragia masiva. Determinar qué pacientes necesitarán intubación orotraqueal o traqueotomía, así como cirugía hemostática, constituye la clave del tratamiento. Conclusiones: La terapia anticoagulante conlleva varias complicaciones que como especialistas ORL deberemos tener en cuenta ante un cuadro clínico de disfagia, disfonía, disnea o signos de hemorragia y conocer las posibilidades de intervención en función de la gravedad de cada caso.

PALABRAS CLAVE hematoma retrofaríngeo; anticoagulantes; trastornos de deglución; disnea; faringe; laringe

SUMMARY Introduction and Objective: Pharyngeal-laryngeal hematoma spontaneous shows the importance of a complete ENT examination before symptoms banal appearance and correct history, in the case presented, it unveiled the therapeutic use of anticoagulants. Case description: A 55 year old woman who come to the emergency by dysphagia, unexplained. The inspection shows the presence of a hematoma in the pharyngeal-laryngeal region after the anticoagulant therapy was reversed, evolved favorably with conservative treatment. Discussion: In this case, 
apart from medical management to perform the hematology department, we focus our therapeutic approach in the protection of the airway and prevent a possible massive bleeding. Determine which patients require endotracheal intubation or tracheostomy and hemostatic surgery, it is the key to treatment. Conclusions: The anticoagulant therapy involves several complications ENT specialists must consider before clinical symptoms of dysphagia, dysphonia, dyspnea or signs of bleeding and know the possibilities of performance depending on the severity of each case.

KEYWORDS

retropharyngeal hematoma; anticoagulants; deglutition disorders; dyspnea; pharynx; larynx

\section{INTRODUCCIÓN}

El hematoma faringolaríngeo es un proceso infrecuente que, en su evolución, puede poner en peligro la vida del paciente [1] (hematoma asfixiante [2]). De causa espontánea (diátesis hemorrágica, sangrado de tiroides o adenoma paratiroideo, anticoagulación, hipertensión arterial, aneurisma de arteria carótida...) o secundaria (infección, tos, traumatismo, daño iatrogénico, ingestión de cuerpo extraño...) [3-6], es difícil de diagnosticar [7]. Aunque clínicamente se describe una triada clásica, no siempre se manifiesta de forma completa [6,8-9]. Finalmente, no existe consenso en relación al tratamiento de la vía aérea y el criterio que se aplica depende de cada caso en particular [1]. El hematoma espontáneo faringolaríngeo por tratamiento anticoagulante es excepcional, aumentando el riesgo a partir de los 65 años al incrementarse los factores locales y el requerimiento de anticoagulantes (patología cardiaca) [4].

Presentamos el caso clínico de una paciente medicada con cumarínicos que desarrolló un hematoma espontáneo faringolaríngeo.

\section{DESCRIPCIÓN}

Mujer de 55 años en tratamiento con anticoagulantes orales por cardiopatía reumática con sustitución valvular con prótesis mecánica 15 años antes. Acudió refiriendo dolor de garganta de 3 días de evolución con leve disnea relacionada con los esfuerzos, sin fiebre y orofaringe normal. En la nasofibroscopia se observó abombamiento de coloración violácea desde la base de la lengua, epiglotis, endolaringe y senos piriformes, con buena luz glótica (Figura 1).

La Tomografía Computarizada (TC) evidenció asimetría por engrosamiento de partes blandas en hipofaringe con obliteración del seno piriforme izquierdo, compatible con hematoma faringolaríngeo, sin signos de sangrado agudo (Figura 1).
EI INR (International Normalized Ratio) estaba en 10 (rango normal de 0,8-1,3 y rango terapéutico para prótesis mecánicas de 2.5-3.5), con hemoglobina de $13 \mathrm{~g} / \mathrm{dl}$ (rango 12-16). El Servicio de Hematología instauró tratamiento para revertir la anticoagulación (con plasma fresco y Vitamina K parenteral).
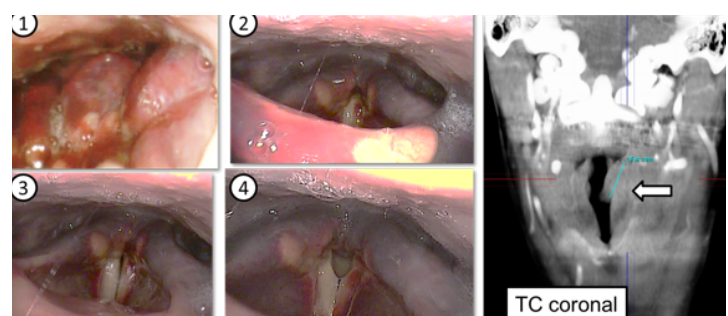

Figura 1. Exploración mediante fibroscopio y TC, al ingreso del paciente. Hematoma de base de lengua (1), equimosis de epiglotis (2), hematoma en seno piriforme izquierdo y equimosis de cuerdas vocales y bandas ventriculares, sobre todo del lado izquierdo (3 y 4). TC coronal con engrosamiento de la pared faríngea izquierda (flecha), que protruye discretamente en la vía aérea.

Se mantuvo a la paciente en observación con controles endoscópicos y fue dada de alta a los 10 días (Figura 2).

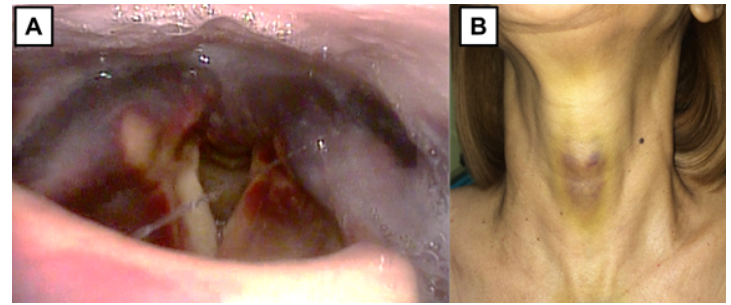

Figura 2. La nasofibroscopia muestra la mejoría parcial del hematoma faringolaríngeo $(A)$, y la aparición de equimosis en la piel anterior del cuello (B), dos días después del alta (12 día).

\section{DISCUSIÓN}

La complicación más frecuente de la terapia anticoagulante es la hemorragia, debiéndose tener en cuenta la sobre-anticoagulación, que puede ser secundaria a patologías asociadas 
o a interacciones medicamentosas $[10,11]$. Aunque no se daban estos supuestos en nuestra paciente, al ser portadora de una válvula cardiaca mecánica se requería mantener unos niveles más altos de INR que en otras circunstancias. Pero superar los valores de 4.5 en el INR, no aumenta el beneficio terapéutico y sin embargo sí lo hace el riesgo de hemorragia [12]. El sangrado espontáneo en los pacientes anticoagulados, suele producirse en el tracto gastrointestinal, el sistema genitourinario o el sistema nervioso central [11]. La incidencia por año de tratamiento es del 4,1-5,2\% [3]. El tracto aerodigestivo superior es una localización muy infrecuente, pudiendo presentarse en los espacios submaxilar, sublingual, peritonsilar [1, 3], siendo excepcional en la región faringolaríngea (unos 12 casos descritos de hematoma espontáneo) $[7,9,13,14]$, que es donde existe el mayor riesgo obstructivo de la vía aérea.

El espacio retrofaríngeo es un área de tejido conectivo laxo situado entre la fascia visceral de la faringe y la capa alar de la fascia cervical profunda. Se extiende desde la base del cráneo hasta donde se fusionan ambas membranas, a nivel de C7-T4, por lo que un sangrado y el hematoma secundario, pueden comprender desde la base del cráneo al mediastino e incluso, dada la comunicación con los espacios parafaríngeos y pretraqueal, hacia estas zonas del cuello [10]. Es probable que el abombamiento retrofaríngeo no sólo se deba a la cantidad de sangre acumulada, sino también al edema secundario que la congestión venosa y linfática generan [15]. Lo que nos compete como especialistas ORL es la sospecha de esta patología en pacientes con clínica de compresión esófago-traqueal. El dolor de garganta suele ser el síntoma inicial, pudiéndose diagnosticar erróneamente de faringitis vírica [6] y en caso de observar una protrusión retrofaríngea, hacernos pensar en un posible absceso [7, 11]. La triada clínica característica [6, 8-9] se constituye ante la presencia de síntomas de compresión traqueal y esofágica (odinofagia, disfagia, sensación de globo faríngeo, voz ronca o de "patata caliente», sialorrea, tos o hemoptisis y disnea), sumados a un desplazamiento ventral de la tráquea en el estudio radiológico y a la presencia de equímosis subcutánea en cuello (Figura 2) y parte superior del tórax. Sin embargo esta tríada no siempre aparece o lo hace tardíamente. El hematoma faringolaríngeo genera un importante dilema terapéutico $[1,16,17]$. Tenemos que elegir entre la observación, la intubación o la traqueotomía, en una patología, cuyo curso natural desencadena con frecuencia una obstrucción respiratoria. En los pacientes con hematoma retrofaríngeo espontáneo donde se puede valorar el tratamiento instaurado, se ve que un $13 \%$ requiere intubación, un $9 \%$ traqueotomía y en un $47 \%$ es suficiente la observación [13]. Si la disnea es leve o inexistente y no hay progresión en el compromiso de la vía aérea, la hospitalización y observación con un tratamiento de soporte puede ser suficiente, como en el caso descrito [1]. Es fundamental revertir la anticoagulación y se debe establecer una estrecha vigilancia en las primeras 48 horas por el riesgo asfíctico, que puede desarrollarse rápidamente. Como tratamiento de mantenimiento además de la analgesia, utilizamos corticoides para reducir el edema añadido y antibióticos para evita la sobreinfección [1]. Cuando el distress respiratorio es más intenso, hay que abordar la vía aérea, lo que viene a ser necesario en 1/3 de casos con una mortalidad del 10\% [17]. La intubación, a veces difícil por la escasa luz y mala visión, puede provocar la ruptura del hematoma, facilitando la hemorragia posterior, aunque de menor importancia si tenemos asegurada la vía aérea [11]. El uso del fibroscopio permite disminuir el traumatismo en su realización. La traqueotomía también favorece el sangrado [4] y es más complicada si se ha alterado la anatomía cervical [7, 10]. En cualquier caso, de acuerdo a la situación clínica y a nuestra experiencia, intentaremos tomar rápidamente una decisión (trasladar al paciente a $\mathrm{UCl}$ o al bloque quirúrgico). Una vez estabilizada la vía aérea, se continuará controlando el hematoma, siendo suficiente en la mayoría de casos la observación y el tratamiento de mantenimiento [1]. La evacuación estaría indicada ante la expansión del hematoma, pudiendo utilizar para su drenaje y aspiración un acceso oral, que posibilita la infección bacteriana o la vía cervical, que presenta mayor morbilidad quirúrgica [11].

El hematoma finalmente, se resolvió en 3-4 semanas.

\section{CONCLUSIONES}

El hematoma espontáneo faringolaríngeo en pacientes anticoagulados es infrecuente y ha de sospecharse en caso de odinofagia, tos o disnea. La observación, reversión de la anticoagulación y si es necesario la intervención urgente para asegurar la vía aérea, constituyen el tratamiento apropiado, que debe individualizarse en cada caso. 


\section{AGRADECIMIENTOS}

Agradecemos el trabajo de revisión que ha servido para mejorar la calidad de nuestro manuscrito.

\section{BIBLIOGRAFÍA}

1. Bloom DC, Timothy H, Keefe MA. Anticoagulation and spontaneous retropharyngeal hematoma. J Emerg Med. 2003;24:389-94.

2. Giroux P, Dufault C, Bernier J. Asphyxiating pharyngolaryngeal hematoma during anticoagulant therapy. Can Med Assoc J. 1967;97:185-6.

3. Boster S, Berjin J. Upper airway obstruction complicating warfarin therapy. Ann Emerg Med. 1983;12:711-4.

4. Garcia FJ, Velert MM. Obstrucción de vías aéreas altas como complicación del tratamiento con acenocumarol. Sangre. 1998;43:223-6.

5. Pino Rivero $V$, Pantoja-Hernández CG González-Palomino A, Pardo-Romero G, Mora-Santos ME, Carrasco-Claver F, et al. Hematoma faringolaríngeo y cervical espon-táneo tras crisis tusígena. An Otorrinolaringol Ibero Am. 2007;34:9-15.

6. Muñoz A, Fischbein NJ, de Vergas J, Crespo J, Alvarez-Vincent J. Spontaneous retropharyngeal hematoma: diagnosis by MR imaging. AJNR Am J Neuroradiol. 2001;22:1209-11.

7. Rosenbaum L, Thurman P, Krantz SB. Upper airway obstruction as a complication of oral anticoagulation therapy. Report of three cases. Arch Intern Med. 1979;139:1151-3

8. Jones T, Owen G, Morar P. Spontaneous retropharyngeal haematoma attributable to Epstein Barr virus infection. J Laryngol Otol. 1996;110:1075-7.
9. Marti L, Grau M, Martinez R. Hematoma retrofaríngeo espontaneo. Acta Otorrinolaringol Esp. 2012;63:77-8.

10. Akoğlu E, Seyfeli E, Akoğlu S, Karazincir $S$, Okuyucu S, Dağli AS. Retropharyngeal hematoma as a complication of anticoagulation therapy. Ear Nose Throat J. 2008;87:156-9.

11. Triaridis $\mathrm{S}$, Tsiropoulos $\mathrm{G}$, Ra-chovitsas D, Psillas G, Vital V. Spontaneous haematoma of the pharynx due to a rare drug interaction. Hippokratia. 2009;13:175-7.

12. Hirsh J, Poller L, Deykin D, Levine M, Dalen J. Optimal therapeutic range for oral anticoagulants. Chest. 1989;95 (Suppl):5s-11s.

13. Paleri V, Maroju RS, Ali MS, Ruckley RW. Spontaneous retro and parapharyngeal haematoma caused by intrathyroid bleed. J Laryngol Otol. 2002;116:854-8.

14. Gao R, Shao A, Dorman B. Spontaneous retropharyngeal haematoma: report of a case. ANZ J Surg. 2011;81:756.

15. Inokuchi G, Kurita N, Baba M, Hata Y, Okuno T. Retropharyngeal hematoma from parathyroid hemorrhage in a hemodialysis patient. Auris Nasus Larynx. 2012;39:527-30.

16. Gurr DE, Walls RM. Anticoagulation and spontaneous retropharyngeal hematoma. J Emerg Med. 2003;24:469-70.

17. Sinert R, Scalea T. Retropharyngeal and bowel hematomas in an anticoagulated patient. Acad Emerg Med. 1994;1:67-72. 